\title{
Vers une approche opérationnelle pour l'évaluation des EIAH
}

\author{
Chieu VU MINH [CLIPS-IMAG, Grenoble, France] \\ Elie MILGROM [INGI-UCL, Louvain-la-Neuve, Belgique]
}

RÉSUMÉ : Le constructivisme est une théorie de l'apprentissage qui postule que l'individu apprend en construisant activement ses propres connaissances, sur la base de ses connaissances préalables, qu'il ajuste et modifie au long de son apprentissage. Plusieurs systèmes d'apprentissage constructiviste basés sur les TIC ont été proposés ces dernières années. Un problème critique relatif à la conception et l'utilisation de ce type de systèmes est la pénurie de moyens pratiques pour évaluer la conformité des systèmes existants avec des principes pédagogiques du constructivisme. Notre objectif de recherche est d'aider à concevoir et à utiliser des environnements d'apprentissage constructiviste qui mettent réellement en œuvre les principes du constructivisme. Pour ce faire, nous avons proposé un ensemble de critères pour certains aspects fondamentaux $\mathrm{du}$ constructivisme et nous les avons utilisés comme un cadre utile pour concevoir et évaluer des environnements d'apprentissage. Dans cet article, nous présentons et discutons trois approches, y compris la nôtre, qui s'appuient sur des critères afin de faciliter l'évaluation des environnements d'apprentissage constructiviste.

— MOTS CLÉS : Constructivisme, environnements d'apprentissage, critères d'évaluation.

ABSTRACT : Constructivism is a learning theory which states that people learn by actively constructing their own knowledge, based on prior knowledge. A significant number of ICT-based constructivist learning systems have been proposed in recent years. A critical problem related to the design and use of this kind of systems has been the lack of practical means for evaluating the actual conformity of such systems with constructivist principles. Our research aims to help designing truly constructivist learning environments. For that purpose, we have proposed a set of operational criteria for certain aspects of constructivism and we have used them as a useful framework both for designing and for analyzing learning environments. In this paper, we present and discuss three approaches, including ours, which are based on criteria to facilitate the evaluation of constructivist learning environments.

KEYWORDS : Constructivism, learning environments, evaluation criteria.

- 1 . Introduction

- $\underline{\text { 2. Les multiples paradigmes du constructivisme }}$

- 3 . Analyse de trois approches basées sur des critères

- 4 . Conclusion

- $\underline{\text { Remerciements }}$

- $\underline{\text { BIBLIOGRAPHIE }}$

\section{Introduction}

\subsection{Environnements d'apprentissage constructiviste}

Le constructivisme (Piaget, 1975) est une approche pédagogique qui "souligne que l'individu apprend mieux s'il construit activement ses connaissances" (Santrock, 2001). L'apprentissage constructiviste est un processus de construction et de transformation des connaissances (Bourgeois et Nizet, 1999). Voici un exemple d'apprentissage constructiviste proposé par Bruner $\underline{(\text { Bruner, 1973) }}$ :

Le concept de nombre premier semble être maîtrisé plus aisément lorsque l'enfant, par la construction, découvre que certains nombres de haricots ne peuvent pas être déposés, un par case, de manière à remplir complètement les lignes et les colonnes d'un tableau. De telles quantités de haricots ne peuvent être déposées que soit dans une seule ligne ou colonne simple, soit dans un tableau dans lequel il manque toujours un ou plusieurs haricots pour obtenir un remplissage complet. Ces nombres, l'enfant l'apprend, sont appelés "nombres premiers"... Il est facile pour l'enfant d'aller de cette étape à la compréhension du fait qu'un tableau "multiple" est un arrangement 
complet de quantités dans des lignes et colonnes multiples. Ce sont les concepts de factorisation, de multiplication et de nombres premiers qui sont visualisés dans cette construction. (p. 428)

Un exemple d'apprentissage non constructiviste serait le cas dans lequel l'enseignant demande à l'enfant de lire et de mémoriser une définition textuelle du concept de nombre premier. Cette situation ne favorise pas l'apprentissage, d'un point de vue constructiviste, parce qu'elle peut amener l'enfant à un apprentissage passif ou "par cœur" : l'enfant n'a pas les moyens de construire lui-même sa propre conception de nombres premiers (Chieu, 2005).

De nombreux chercheurs en sciences de l'éducation soutiennent le principe central du constructivisme présenté plus haut. Cependant, il existe plusieurs théories constructivistes de l'apprentissage et de l'enseignement (Driscoll, 2000). Les chercheurs en sciences de l'éducation, en psychologie de l'apprentissage, en didactique et en sciences des technologies de l'information et de la communication pour l'apprentissage humain ont développé une multitude d'aspects du constructivisme. Ces variantes incluent l'apprentissage "génératif" (generative learning) (CTGV, 1991), (Wittrock, 1985), l'apprentissage par découverte (Bruner, 1986), la cognition distribuée (Salomon, 1994), la théorie de la flexibilité cognitive (Spiro et al., 1991). Le constructivisme n'est qu'un des termes utilisés pour décrire ces variantes.

De plus, ces dernières années, l'approche pédagogique constructiviste a été adoptée par un nombre important de concepteurs d'environnements informatiques qui affirment favoriser explicitement l'apprentissage constructiviste (Kinshuk et al., 2004). Nous constatons cependant le problème suivant en ce qui concerne ces environnements informatiques pour l'apprentissage constructiviste : alors qu'existent de nombreuses descriptions des principes pédagogiques du constructivisme, nous n'avons trouvé que très peu de moyens pratiques pour aider les concepteurs, chercheurs et praticiens à évaluer effectivement leur propres systèmes d'apprentissage selon des principes constructivistes (Driscoll, 2000), (Jonnaert et Vander Borght, 2003), (Jonassen et Rohrer-Murphy, 1999).

En effet, si beaucoup de chercheurs en sciences de l'éducation tendent à accepter l'hypothèse centrale du constructivisme présentée plus haut, ces mêmes chercheurs ont proposé un grand nombre de différentes implications pédagogiques à partir des mêmes principes de base. Par exemple, le Cognition and Technology Group de l'Université Vanderbilt (CTGV, 1991) et Jonassen (Jonassen, 1999) ont soutenu que l'apprenant doit nécessairement faire face à des situations complexes pour développer ses compétences en résolution de problèmes. Spiro et ses collègues (Spiro et al., 1991), qui s'intéressent à la flexibilité cognitive, ont souligné : "revoir la même matière, à différents moments, dans des contextes réarrangés, pour différents objectifs, et selon différentes perspectives conceptuelles, est essentiel pour atteindre les objectifs de l'acquisition de connaissances avancées". Bourgeois et Nizet (Bourgeois et Nizet, 1999) ont exprimé le point de vue que la négociation sociale est nécessaire pour que l'étudiant comprenne les points de vue de ses pairs. Reeves et Okey (Reeves et Okey, $1996)$ et Shepard (1991) ont argumenté en faveur de méthodes d'évaluation telles que l'interrogation, l'observation et la réalisation de tâches "réalistes" (par exemple : demander à l'apprenant d'écrire un essai, de conduire une expérimentation ou de réaliser un projet). Toutes ces indications sont certainement de nature à favoriser l'apprentissage constructiviste, mais, de notre point de vue, elles sont trop générales pour que les concepteurs, chercheurs et praticiens puissent imaginer concrètement comment concevoir ou évaluer des environnements d'apprentissage constructiviste.

\subsection{Contributions}

Notre objectif de recherche est d'aider les concepteurs, chercheurs et praticiens à concevoir et évaluer des environnements qui favorisent un apprentissage véritablement constructiviste. Pour cela, nous devons clarifier ce que le constructivisme entraîne de manière opérationnelle. À cet effet, nous proposons un ensemble de critères pour certains aspects majeurs du constructivisme et nous utilisons ces critères comme un cadre qui facilite la conception et l'évaluation des environnements d'apprentissage constructiviste, ou plus précisément, pour cerner les conditions qui favorisent l'apprentissage constructiviste. Nous définissons une condition d'apprentissage comme une ressource d'information, un dispositif pédagogique, un dispositif d'évaluation ou un moyen de communication qui facilite la poursuite d'un objectif d'apprentissage par l'apprenant.

Dans cet article, afin de valider notre hypothèse que l'approche opérationnelle est effective pour l'évaluation des environnements d'apprentissage constructiviste, nous analysons et discutons trois approches (y compris la nôtre) qui s'appuient sur des critères en vue de faciliter l'évaluation des conditions soutenant l'apprentissage constructiviste.

\subsection{Structure de l'article}

Dans la section 2, nous introduisons une variété de paradigmes du constructivisme. Dans la section 3, nous présentons notre analyse de trois approches basées sur des critères pour l'évaluation des conditions d'apprentissage constructiviste. Dans la section finale, nous tirons de notre analyse plusieurs conclusions pour la conception et l'évaluation des environnements informatiques pour l'apprentissage humain (EIAH). 


\section{Les multiples paradigmes du constructivisme}

Considérant les différentes variantes du constructivisme, les chercheurs dans le domaine de la psychologie de l'apprentissage, par exemple (Bourgeois et Frenay, 2002) ou (Santrock, 2001), ont classifié les approches constructivistes en deux paradigmes principaux : (a) une approche constructiviste cognitive fondée principalement sur les travaux de Piaget (Piaget, 1975) et (b) un nombre d'approches socioconstructivistes fondées, par exemple, sur les travaux de Bruner (Bruner, 1996), Doise et Mugny (Doise et Mugny, 1997) ou encore Vygotsky (Vygotsky, 1962). Les tenants du premier paradigme postulent que l'apprenant construit de nouvelles connaissances en transformant, organisant et réorganisant ses propres connaissances préalables. Les chercheurs qui préfèrent le deuxième paradigme postulent que l'individu construit des connaissances grâce aux interactions sociales avec d'autres personnes. La différence conceptuelle entre les deux paradigmes est la différence entre le développement individuel et la collaboration et l'interaction sociale (Rogoff, 1998). Cette affirmation ne signifie pas que le constructivisme cognitif néglige l'interaction sociale ou que le socioconstructivisme ignore le développement individuel : les deux paradigmes tiennent compte des deux aspects mais avec différentes emphases (Bourgeois et Nizet, 1999). En effet, le socioconstructivisme se focalise davantage sur la question suivante, à laquelle le constructivisme cognitif n'attache que peu d'importance : dans quelles conditions et selon quelles modalités l'interaction sociale favorise-t-elle l'apprentissage ? Dans ce sens, on peut affirmer que le socioconstructivisme n'est pas une alternative au constructivisme, mais plutôt une variante qui attache une importance particulière aux interactions sociales.

Examinons, pour commencer, deux questions majeures qui ont préoccupé les psychologues de l'apprentissage : qu'est-ce que connaître? Qu'est-ce qu'apprendre?

En épistémologie, du point de vue de Piaget, la connaissance n'est pas là, en dehors, externe à l'individu et en attente d'être acquise. Elle n'est pas non plus complètement préformée dans le cerveau de l'individu et prête à émerger lorsque l'individu se développe. Au contraire, la connaissance est inventée et réinventée tout au long du développement de l'individu par les interactions de celui-ci avec le milieu qui l'entoure. Pour renforcer ce point de vue, on peut utiliser l'exemple proposé par Kuhn (1983) et cité dans Jonnaert et Vander Borght (2003) :

Désirant connaître ce que représente, pour des scientifiques, la théorie atomique, un chercheur nord-américain interroge deux chercheurs reconnus par la communauté scientifique internationale dans leur domaine respectif: la chimie et la physique. Il leur demande si un atome d'hélium est ou n'est pas une molécule. La réponse de l'un et l'autre ne s'accorde pas. Pour le chimiste, l'atome d'hélium est une molécule. Ce dernier argumente sa réponse en faisant référence à la théorie cinétique des gaz. Pour le physicien, l'atome d'hélium n'est pas une molécule. Ce dernier argumente sa réponse en affirmant qu'il ne peut en apercevoir le spectre moléculaire. (p. 23)

Ces réponses ne sont pas contradictoires. Une telle situation est fréquente (voir d'autres exemples dans_Chieu, 2005). Chacun, le chimiste et le physicien, construit sa définition d'un atome d'hélium en fonction de son champ de référence. Un scientifique peut faire coexister simultanément les deux définitions dans sa "tête".

Alors que le premier paradigme (constructivisme cognitif) se focalise avant tout sur l'acte de connaître chez le sujet individuel dans ses interactions avec son environnement, le deuxième (socioconstructivisme) appréhende la connaissance essentiellement comme le produit d'un groupe social, d'une communauté engagée dans une culture et des pratiques communes.

Pour ce qui est de l'apprentissage, Piaget s'intéresse avant tout aux mécanismes d'interactions cognitives du sujet avec son environnement ici et maintenant. Le socioconstructivisme met un accent supplémentaire sur une composante particulière de l'environnement : les autres apprenants avec lesquels chaque apprenant interagit.

Le processus d'apprentissage dans le constructivisme piagétien s'articule autour de deux mécanismes indissociables : l'assimilation et l'accommodation. On peut les décrire très schématiquement comme suit (Bourgeois et Frenay, 2002) :

a. Assimilation: Au départ, l'individu active un certain nombre de structures cognitives dont il dispose dans le répertoire enregistré en mémoire et qu'il va utiliser pour traiter l'information à laquelle il est confronté dans une situation donnée. Dans l'exemple de l'apprentissage des nombres premiers présenté dans la section 1.1, lorsque l'on donne à l'enfant 12 haricots, l'enfant active ses connaissances préalables de la factorisation et de la multiplication afin de mettre les haricots dans un tableau complet de lignes et de colonnes (par exemple : 3 lignes de 4 colonnes).

b. Accommodation: Il peut arriver, en cours de traitement, que les structures mobilisées s'avèrent inadéquates pour traiter l'information. Emerge dans ce cas ce que Piaget appelle un conflit cognitif, une perturbation des structures mobilisées, que l'individu va dès lors s'efforcer de dépasser, de résoudre. Il peut le faire de deux manières : soit, en utilisant des stratégies qui, au bout du compte, permettent de résoudre le conflit tout en préservant intactes les structures cognitives dans leur état initial, soit en transformant les structures initiales en structures nouvelles permettant de prendre en compte l'information initialement incompatible. Pour Piaget, ce 
n'est que dans ce dernier cas que l'on peut parler d'apprentissage. Par exemple, dans l'apprentissage des nombres premiers, lorsqu'on donne 11 haricots à l'enfant, un conflit cognitif peut se produire chez lui car il n'arrive pas à déposer 11 haricots dans un tableau complet de lignes et de colonnes "multiples" ( 2 x 6 ou 3 x 4). Ce conflit cognitif motive donc l'enfant à trouver une solution pour le surmonter. Il peut ainsi, par exemple, transformer les structures initiales (la factorisation et la multiplication) en structures nouvelles (la reconnaissance de la différence entre deux groupes de nombres : le nombre 12 appartient au premier groupe et le nombre 11 appartient au deuxième). Les nombres du second groupe, l'enfant l'apprend ensuite, sont des nombres premiers.

L'assimilation et l'accommodation sont indissociables, car une tentative insatisfaisante d'assimiler une nouvelle information dans les structures cognitives existantes peut avoir comme conséquence un certain ajustement de ces structures cognitives (ainsi, accommoder l'information) et, réciproquement, une telle accommodation affecte une assimilation suivante, qui procédera maintenant en accord avec la nouvelle structure. De plus, l'assimilation et l'accommodation constituent ensemble le processus principal de développement : l'équilibration. Ce processus caractérise la transition de l'individu d'un stade de développement au stade de développement suivant. Par exemple, l'évolution d'un enfant qui peut résoudre des problèmes concrets dans un mode logique à un adulte qui peut résoudre des problèmes abstraits dans un mode systématique et logique. A travers la trajectoire de sa vie, un individu rencontre sans cesse des expériences "anormales" qui créent des états de déséquilibre. L'individu ne peut résoudre un état de déséquilibre qu'en adoptant un mode plus adaptatif et plus sophistiqué de pensée. Dans ce cas, l'individu atteint un nouvel équilibre : c'est l'apprentissage.

Piaget a noté que l'équilibration se produit dès la naissance d'un nouveau-né. Ce dernier nait avec un certain nombre de réflexes innés (des structures cognitives primitives), par exemple : la succion, la réaction aux bruits. En peu de temps, il commence à modifier ces réflexes pour les rendre plus adaptatifs : la succion d'un doigt, par exemple, devient une action différente de la succion d'un mamelon.

Dans la perspective socioconstructiviste, l'apprentissage est fondamentalement abordé comme le processus par lequel le savoir circule, se construit et se transforme au centre d'un groupe social, d'une communauté. Dans cette perspective, apprendre, pour le sujet individuel, c'est participer à ce processus collectif et collaboratif de co-construction du savoir, c'est s'inscrire dans une relation avec d'autres membres du groupe qui promeut cette co-construction : c'est en participant que l'on apprend et réciproquement. L'attention est donc portée en priorité à la connaissance comme un processus et un produit essentiellement sociaux, collectifs et collaboratifs, propres à un groupe. Les processus individuels de connaissance et de pensée sont pris en compte, mais toujours comme situés dans un contexte donné, c'est-à-dire dans le cadre des interactions du sujet avec les autres membres de la communauté à laquelle il appartient. C'est pour cela qu'on s'intéressera autant à la manière dont l'individu participe et contribue à la construction et à l'usage des connaissances collectives propres à son groupe, qu'à la manière dont le contexte dans lequel le sujet est inséré et les interactions sociales conditionnent ses processus cognitifs individuels (Bourgeois et Frenay, 2002).

Par exemple, pour rendre compte du mécanisme qui préside au développement cognitif, c'est-à-dire au passage d'un stade à l'autre, l'approche historico-culturelle de Vygotski souligne à la fois le rôle central d'autrui avec lequel l'individu interagit et celui de ce qu'il appelle la zone proximale de développement, qui est délimitée, d'un côté, par la tâche la plus difficile que l'individu peut réaliser tout seul, sans aucune aide et, de l'autre côté, par la tâche la plus difficile que le sujet peut réaliser avec l'aide d'autres. En d'autres termes, si l'intelligence peut se développer, c'est parce que, d'une part, l'individu interagit avec un environnement social qui peut lui apporter l'aide dont il a besoin et, d'autre part, parce qu'il est prêt à tirer profit de celle-ci. Donc, pour Vygotski, la question centrale pour l'enseignant est d'identifier la zone proximale de développement de l'apprenant dans tel ou tel domaine d'activité et les modalités de relation les plus favorables à l'apprentissage dans cette zone (Bourgeois et Frenay, 2002).

Bien qu'il n'existe aucun ensemble précis de pratiques d'enseignement qui constitue de manière univoque une approche constructiviste ou socioconstructiviste, beaucoup de chercheurs en éducation ont suggéré une variété de principes pédagogiques conformes aux principes du (socio)constructivisme. Dans la section suivante, nous illustrons en détail comment les chercheurs dans différentes disciplines interprètent le (socio)constructivisme en vue de définir et d'évaluer les conditions qui, selon eux, soutiennent l'apprentissage constructiviste. Dans la suite, lorsque nous utiliserons le terme "constructivisme" sans plus le qualifier, nous désignerons à la fois le constructivisme cognitif et le socioconstructivisme.

\section{Analyse de trois approches basées sur des critères}

Dans cette section-ci, nous présentons notre analyse de trois approches qui sont basées sur des critères pour évaluer des conditions d'apprentissage constructiviste. Pour réaliser une telle analyse de manière pertinente, il faudrait, de notre point de vue, prendre en compte les rubriques suivantes :

a. Approche pédagogique: Le constructivisme comporte beaucoup de variantes (Driscoll, 2000). Pour 
comprendre le travail réalisé par les auteurs, il faut donc comprendre le paradigme qui guide l'ensemble de la réflexion qu'ils proposent (Jonnaert et Vander Borght, 2003).

b. Critères: Nous étudions quels critères les auteurs définissent et pourquoi ils les proposent.

c. Application : Nous examinons comment les auteurs appliquent leurs propres critères pour la pratique de l'évaluation des conditions d'apprentissage.

d. Discussion : Sur la base de notre compréhension de l'ensemble des critères proposés, nous discutons l'utilité de ces critères pour la pratique.

e. Validation : Nous examinons quelles expériences les auteurs ont réalisées pour valider la pertinence de leurs critères dans des situations réelles d'enseignement. La question majeure est : est-ce que les conditions qui satisfont l'ensemble des critères favorisent véritablement l'apprentissage constructiviste chez un apprenant ?

Par la suite, nous utilisons ces rubriques afin d'analyser des critères proposés par Brousseau et Vázquez-Abad (Brousseau et Vázquez-Abad, 2003) dans la section 3.1, par Jonnaert et Vander Borght (Jonnaert et Vander Borght, 2003) dans la section 3.2 et par nous-mêmes (Chieu, Milgrom et Frenay, 2004), (Chieu et Milgrom. $\underline{2005)}$, (Chieu, 2005) dans la section 3.3. La section 3.4 est consacrée à la validation des approches. Notre analyse a été réalisée en examinant des documents publiés par les auteurs. Si l'analyse de notre propre approche est plus élaborée que celle de deux autres, c'est parce que nous avons proposé un plus grand nombre de critères que les auteurs de la première approche et parce que nous avons appliqué nos critères pour construire un EIAH concret, ce qui n'est pas le cas pour les auteurs de l'approche SCI.

\subsection{Critères proposés par Brousseau et Vázquez-Abad}

Brousseau et Vázquez-Abad sont didacticiens à l'Université de Montréal. Ils ont examiné un certain nombre de perspectives constructivistes et ont retenu certaines caractéristiques essentielles du constructivisme en vue d'analyser un exemple de travail et d'apprentissage collaboratif s'appuyant sur les TIC. Ils ont conclu qu'une activité telle que celle de leur projet TACTICS peut être considérée comme étant de nature constructiviste.

\subsubsection{Approche pédagogique}

Les deux auteurs n'ont pas suivi une perspective constructiviste unique pour l'évaluation de leur environnement d'apprentissage. Par contre, ils ont examiné une pluralité de points de vue constructivistes pour proposer une série de critères essentiels, selon eux, pour garantir le constructivisme.

Ils s'appuient principalement et explicitement sur les travaux de Piaget présentés dans la section 2. Ils adhèrent au point de vue d'Astolfi (Astolfi, 1989) qui affirme que le constructivisme consiste en un ensemble de procédures d'enseignement qui mettent l'étudiant au cœur de ses apprentissages. En opposition à l'approche par transmission des connaissances, les apprentissages doivent toujours être construits et reconstruits activement par l'apprenant lui-même. Cependant, cela ne signifie nullement que l'enseignant n'a pas un rôle central dans l'apprentissage. Au contraire, les apprentissages doivent être orchestrés par l'enseignant dans le cadre d'un modèle didactique où l'apprenant devient un acteur essentiel de son savoir et qui le conduit à des ruptures épistémologiques et à des dépassements intellectuels.

Brousseau et Vázquez-Abad affirment, en outre, qu'il est pertinent de prendre également en compte les concepts $\mathrm{du}$ socioconstructivisme, qui met en avant le rôle des interactions sociales et des collaborations dans la construction de savoirs chez l'apprenant (Wallon, 1941), (Vygotsky, 1962). D'un point de vue socioconstructiviste, une nouvelle connaissance est d'abord subjective, propre à un individu, et devient objective, commune à un groupe, seulement lorsqu'elle est rendue publique et qu'elle est acceptée socialement. La dimension "socio" du socioconstructivisme permet donc à l'apprenant de donner du sens aux connaissances qu'il construit (Ernest, 1991). Dans la section 3.1.3, nous verrons comment Brousseau et Vázquez-Abad organisent la négociation sociale chez les élèves en vue de favoriser l'apprentissage.

\subsubsection{Critères}

Après avoir examiné un certain nombre de perspectives constructivistes, Brousseau et Vázquez-Abad ont défini trois critères qui, selon eux, expriment les points communs majeurs de ces perspectives :

a. Le constructivisme n'admet pas qu'une connaissance puisse être transmise d'une personne à une autre.

b. Le savoir reconnu est un savoir pratique permettant à l'apprenant de survivre, de résoudre des problèmes et de réaliser des projets.

c. Les processus d'apprentissage doivent être centrés sur l'apprenant et le conduire de façon individuelle et collective à la construction de connaissances.

Les deux auteurs ont ensuite utilisé ces trois critères comme un guide afin d'évaluer la nature constructiviste 
d'une activité pédagogique (ou, plus spécifiquement, des conditions d'apprentissage dans cette activité), comme présenté dans la sous-section qui suit.

\subsubsection{Application}

Le projet TACTICS (Travail et Apprentissage Collaboratifs avec les Technologies de l'Information et des Communications) (Vázquez-Abad et al., 2003) a été conçu pour mettre en place une communauté visant à promouvoir l'apprentissage collaboratif réparti des sciences au niveau de l'enseignement secondaire. Depuis l'année scolaire 2000-2001, le projet rassemble une communauté composée de quelques centaines de chercheurs, d'étudiants diplômés, d'enseignants et de leurs élèves de quinze à dix-huit ans qui sont répartis géographiquement au Mexique et à Montréal.

Le développement du projet s'appuie sur le modèle du casse-tête en apprentissage collaboratif (Slavin, 1995), modifié pour l'utilisation en mode réparti (Winer et al., 2000). Ce modèle propose la division des apprentissages visés en une série de regroupements équilibrés, complémentaires et essentiels de manière à distribuer la responsabilité de ces apprentissages et à créer la nécessité d'une concertation et d'une synthèse pour arriver au résultat désiré.

Les élèves de TACTICS sont organisés en équipes de base, elles-mêmes formées de trois sous équipes (dites d'experts) et regroupant trois ou quatre apprenants chacune. L'objectif d'apprentissage des élèves est de mener une recherche d'information et de produire une synthèse commune sur un thème scientifique qu'ils auront choisi dans une liste de thèmes préalablement établie par les enseignants et les chercheurs de la communauté de TACTICS. Ces thèmes sont tous subdivisés en trois sujets qui sont distribués à chacune des équipes d'experts et dont elles sont responsables.

\begin{tabular}{|c|c|c|}
\hline Activité & Description & Analyse \\
\hline $\begin{array}{l}\text { Présentation de tous } \\
\text { les participants }\end{array}$ & Présentation personnelle aux coéquipiers & $\begin{array}{l}\text { Création d'un lien affectif } \\
\text { entre participants }\end{array}$ \\
\hline $\begin{array}{l}\text { Travail en équipe } \\
\text { d'experts sur un des } \\
\text { trois sujets du thème }\end{array}$ & $\begin{array}{l}\text { Recherches sur Internet, à la bibliothèque, auprès de parents, etc. } \\
\text { pour produire conjointement un travail sur un des trois sujets du } \\
\text { thème; échange avec d'autres équipes d'experts ayant le même } \\
\text { sujet de recherche }\end{array}$ & $\begin{array}{l}\text { Construction de } \\
\text { connaissances à la fois } \\
\text { individuelle et collective }\end{array}$ \\
\hline $\begin{array}{l}\text { Résumé du travail de } \\
\text { l'équipe d'experts et } \\
\text { préparation des } \\
\text { questions }\end{array}$ & $\begin{array}{l}\text { Résumé du travail précédent et préparation des questions pour } \\
\text { susciter des discutions de deux autres équipes d'experts dans } \\
\text { l'équipe de base }\end{array}$ & $\begin{array}{l}\text { Organisation de } \\
\text { connaissances, discussion } \\
\text { entre pairs, production de } \\
\text { résumés }\end{array}$ \\
\hline $\begin{array}{lr}\text { Lecture des } & \text { résumés, } \\
\text { réponses } & \text { aux } \\
\text { questions } & \text { et } \\
\text { correction } & \end{array}$ & $\begin{array}{l}\text { Travail en groupe pour comprendre des résumés, répondre aux } \\
\text { questions et corriger des réponses de deux autres équipes } \\
\text { d'experts }\end{array}$ & $\begin{array}{l}\text { Construction de } \\
\text { connaissances } \\
\text { individuelle, sociale et } \\
\text { collective }\end{array}$ \\
\hline $\begin{array}{l}\text { Préparation de la } \\
\text { synthèse du thème de } \\
\text { l'équipe de base }\end{array}$ & $\begin{array}{l}\text { Pour chacune des équipes de base, à partir des trois résumés } \\
\text { produits, une première équipe d'experts note les similitudes entre } \\
\text { les trois sujets du thème de recherche, une seconde en note les } \\
\text { différences et une troisième rédige une ébauche de la synthèse du } \\
\text { thème en s'appuyant sur les travaux réalisés par les deux } \\
\text { premières }\end{array}$ & $\begin{array}{l}\text { Renforcement de la } \\
\text { collaboration entre les } \\
\text { trois équipes d'experts }\end{array}$ \\
\hline $\begin{array}{l}\text { Discussion et } \\
\text { rédaction finale de la } \\
\text { synthèse du thème }\end{array}$ & $\begin{array}{l}\text { Pour remettre la synthèse du thème au public, les trois équipes } \\
\text { d'experts retravaillent ensemble l'ébauche de la synthèse (réviser, } \\
\text { corriger, améliorer et approuver) }\end{array}$ & $\begin{array}{l}\text { Négociation et } \\
\text { discussions pour parvenir } \\
\text { à la synthèse finale }\end{array}$ \\
\hline $\begin{array}{l}\text { Production de la page } \\
\text { Web }\end{array}$ & $\begin{array}{l}\text { Production d'une page Web (publique) qui contient l'information } \\
\text { des trois travaux des équipes d'experts, de leurs trois résumés et } \\
\text { de la synthèse finale de l'équipe de base }\end{array}$ & $\begin{array}{l}\text { Transformation } \\
\text { connaissances }\end{array}$ \\
\hline
\end{tabular}

\section{Tableau 1 : Analyse de TACTICS}

Brousseau et Vázquez-Abad utilisent leurs trois critères pour analyser les conditions d'apprentissage créées pendant le travail des élèves, qui est subdivisé en sept parties et qui s'échelonne sur près de sept mois. Nous 
résumons le résultat de cette analyse dans le Tableau 1.

\subsubsection{Discussion}

Brousseau et Vázquez-Abad ont examiné une variété de perspectives constructivistes dans les deux paradigmes principaux présentés dans la section 2 : le constructivisme cognitif et le socioconstructivisme. Ils affirment choisir le constructivisme cognitif pour proposer leurs trois critères (il n'y a que le troisième critère qui aborde la construction collective de connaissances). Cependant, il nous semble que, dans le cadre du projet TACTICS, les deux auteurs se focalisent sur le socioconstructivisme : nous voyons apparaître la collaboration, la négociation et les discussions plus souvent que le développement individuel dans l'activité pédagogique mise en œuvre dans TACTICS.

Les deux auteurs ont montré que, à la lumière de leurs trois critères, les conditions d'apprentissage du projet TACTICS peuvent être considérées comme étant de nature constructiviste. Nous estimons, quant à nous, qu'il faudrait clarifier les trois critères de manière plus opérationnelle en vue de permettre une analyse plus précise et approfondie que celle des deux auteurs.

En effet, les trois critères sont encore trop généraux : il est difficile de les appliquer pour évaluer des conditions d'apprentissage dans des situations d'enseignement quelconques. Par exemple, nous trouvons difficile d'utiliser les trois critères de Brousseau et de Vázquez-Abad pour évaluer l'activité pédagogique de notre propre environnement d'apprentissage (voir la section 3.3.3). Même dans leur analyse, il nous semble qu'il est difficile pour le lecteur de reconnaître la pertinence des trois critères par rapport aux conditions d'apprentissage fournies par le projet TACTICS : on ne voit pas explicitement quelles conditions d'apprentissage satisfont quels critères et comment la satisfaction des critères est justifiée. A titre d'exemple, quel(s) est(sont) le(s) critère(s) satisfait(s) par la deuxième activité à propos de la production d'un travail sur un des trois sujets du thème et comment est(sont)-il(s) satisfait(s)?

Dans les sections suivantes, nous analyserons deux ensembles des critères opérationnels, proposés par Jonnaert et Vander Borght et par nous-mêmes.

\subsection{Critères proposés par Jonnaert et Vander Borght}

Jonnaert est professeur titulaire à l'Université de Sherbrooke ; ses recherches portent sur l'apprentissage et les processus de compréhension et de construction des connaissances mathématiques. Vander Borght est professeur à l'Université catholique de Louvain; ses recherches portent sur la didactique des sciences. Partant d'une approche socioconstructiviste et interactive, les deux auteurs ont proposé une grille qui peut être utilisée pour analyser différentes définitions du concept de l'apprentissage (Jonnaert et Vander Borght, 2003).

\subsubsection{Approche pédagogique}

Les deux auteurs présentent une perspective socioconstructiviste et interactive (le modèle SCI) dans un contexte scolaire. Ce paradigme épistémologique se compose des trois dimensions interdépendantes suivantes :

a. La dimension constructiviste cognitive : Selon les deux auteurs, la perspective constructiviste s'appuie sur le double postulat suivant : (a) l'individu construit ses connaissances à travers sa propre activité ; (b) l'objet manipulé au cours de cette activité n'est autre que sa propre connaissance. Ce double postulat suppose donc, de la part de l'individu qui construit ses connaissances, une activité réflexive sur ses propres connaissances. Cette activité réflexive a pour fonction l'adaptation des connaissances de l'individu aux exigences de la situation à laquelle il est confronté.

b. La dimension "socio" liée aux interactions sociales: Dans un contexte scolaire, les interactions sociales constituent une composante importante de l'apprentissage : Jonnaert et Vander-Borght postulent que l'élève construit ses propres connaissances dans et par les interactions avec les autres. Ces interactions sociales ont aussi une double dimension : (a) il s'agit d'une part des interactions entre pairs ; (b) il s'agit d'autre part des interactions entre l'apprenant et l'enseignant.

c. La dimension "interactive" liée aux interactions avec le milieu : Les apprentissages sont certes des processus individuels qui se développent grâce aux interactions avec les autres, mais aussi grâce aux échanges que l'individu établit avec le milieu qui l'entoure. Ce volet s'appuie sur le postulat suivant : les apprentissages scolaires ne peuvent se vivre qu'en situation; les situations sont à la fois source et critère des connaissances. La situation à laquelle l'apprenant est confronté est source d'apprentissage, car elle confronte les connaissances de l'apprenant aux exigences de la situation. Elle en est aussi critère, car l'apprenant ne peut être efficace dans cette situation que si ses connaissances sont pertinentes.

\subsubsection{Critères}

Pour clarifier le concept de l'apprentissage dans leur modèle SCI, Jonnaert et Vander Borght ont proposé un ensemble de critères opérationnels (Tableau 2) selon les trois dimensions précédemment définies. 


\subsubsection{Application}

Pour montrer l'utilité de leurs critères, les auteurs les ont appliqués pour analyser un certain nombre de définitions du concept de l'apprentissage dans la littérature. Nous illustrons une de ces analyses (Jonnaert et Vander Borght, 2003, p. 261) dans le Tableau 2.

Définition : "(...) L'apprentissage est un processus ; la modification d'acquis antérieurs fait partie intégrante de ce processus ; c'est l'apprenant qui est le principal acteur de l'apprentissage ; un apprentissage scolaire doit s'inscrire dans un contexte porteur de significations pour celui qui apprend ; la logique de celui qui apprend prime sur celle de la matière à enseigner.

(...) L'apprentissage se réalise essentiellement à travers les conduites, les opérations ou les interventions de l'apprenant lui-même ; l'élève réalise personnellement un certain nombre de démarches pour s'approprier de nouvelles conduites et des représentations d'objet, ou pour en changer ; l'apprentissage se construit sur et avec les connaissances antérieures de l'élève ; le processus d'apprentissage évoqué est strictement sous la responsabilité de l'élève qui apprend ; l'enseignant ne contrôle que partiellement la 'situation' dans la quelle il place l'élève ; l'enseignant n'est pas maître de l'apprentissage d'un autre, il contrôle simplement certaines conditions dans lesquelles il place l'élève" (Jonnaert, 1995, p. 39) 


\begin{tabular}{|c|c|c|c|}
\hline Dimensions & Critères & Analyse & Commentaires \\
\hline \multirow[t]{3}{*}{$\begin{array}{l}(1) \\
\text { Constructiviste }\end{array}$} & (1.1) Qui est l'acteur de l'apprentissage? & L'apprenant & $\begin{array}{l}\text { L'apprenant est le principal acteur, } \\
\text { par ailleurs, c'est lui qui contrôle le } \\
\text { processus d'apprentissage. }\end{array}$ \\
\hline & $\begin{array}{l}\text { (1.2) L'apprenant réalise-t-il ses apprentissages } \\
\text { sur base de ses propres connaissances? }\end{array}$ & Oui & $\begin{array}{l}\text { Cette dimension est explicite dans } \\
\text { la définition. }\end{array}$ \\
\hline & $\begin{array}{l}\text { (1.3) L'apprentissage a-t-il du sens pour } \\
\text { l'apprenant? }\end{array}$ & Oui & $\begin{array}{l}\text { Cette dimension est explicite dans } \\
\text { la définition. }\end{array}$ \\
\hline \multirow[t]{3}{*}{ (2) Socio } & $\begin{array}{l}\text { (2.1) L'apprenant réalise-t-il ses apprentissages } \\
\text { en interaction avec ses pairs? }\end{array}$ & non évoqué & \\
\hline & $\begin{array}{l}\text { (2.2) L'apprenant réalise-t-il ses apprentissages } \\
\text { en interaction avec l'enseignant? }\end{array}$ & implicite & $\begin{array}{l}\text { L'enseignant contrôle certaines des } \\
\text { conditions dans lesquelles il place } \\
\text { l'élève. }\end{array}$ \\
\hline & $\begin{array}{l}\text { (2.3) Des zones de dialogue sont-elles définies } \\
\text { pour permettre les interactions entre les } \\
\text { apprenants, l'enseignant et } \\
\text { d'apprentissage? }\end{array}$ & non évoqué & \\
\hline \multirow[t]{5}{*}{ (3) Interactive } & $\begin{array}{l}\text { (3.1) L'apprentissage est-il réalisé à partir de } \\
\text { situations? }\end{array}$ & Oui & $\begin{array}{l}\text { L'apprentissage doit se faire en } \\
\text { contexte } \quad \text { signifiant } \\
\text { l'apprenant. }\end{array}$ \\
\hline & $\begin{array}{l}\text { (3.2) L'apprenant doit-il découvrir l'objet } \\
\text { d'apprentissage dans ces situations? }\end{array}$ & non évoqué & \\
\hline & $\begin{array}{l}\text { (3.3) L'apprenant doit-il interagir avec ces } \\
\text { situations et l'objet d'apprentissage? }\end{array}$ & non évoqué & \\
\hline & $\begin{array}{l}\text { (3.4) La définition [du concept de } \\
\text { l'apprentissage }] \text { permet-elle d'établir une } \\
\text { distinction entre l'objet d'apprentissage et les } \\
\text { connaissances de l'apprenant? }\end{array}$ & Oui & $\begin{array}{l}\text { La logique de l'apprenant est } \\
\text { dissociée de celle de l'objet } \\
\text { d'apprentissage. }\end{array}$ \\
\hline & $\begin{array}{l}\text { (3.5) Existe-t-il des interactions entre l'objet } \\
\text { d'apprentissage et les connaissances de } \\
\text { l'apprenant? }\end{array}$ & non évoqué & \\
\hline
\end{tabular}

Tableau 2 : Analyse d'une définition de l'apprentissage constructiviste

La conclusion tirée par les auteurs est qu'aucune des définitions analysées ne satisfait la totalité des critères qu'ils ont proposés. Ils ont donc proposé leur propre définition du concept d'apprentissage, qui satisfait l'ensemble des critères (c'est-à-dire "apprenant" pour le critère 1.1 et "oui" pour les autres critères). Voici le résumé de la définition :

Un apprentissage est un processus dynamique par lequel un apprenant, à travers une série d'échanges avec ses pairs et l'enseignant, met en interaction ses connaissances avec des savoirs dans l'objectif de créer de nouvelles connaissances adaptées aux contraintes et aux ressources de la situation à laquelle il est actuellement confronté dans l'objectif d'utiliser ses nouvelles connaissances dans des situations non didactiques. (p. 266)

\subsubsection{Discussion}

Jonnaert et Vander Borght ont proposé leurs critères selon une perspective socioconstructiviste et interactive. Bien que les auteurs n'utilisent leurs critères que pour analyser des définitions du concept de l'apprentissage, nous pensons que ces critères sont suffisamment applicables et opérationnels pour permettre d'évaluer des conditions d'apprentissage dans une variété de contextes d'apprentissage, comprenant celui des EIAH. En effet, pour examiner le caractère opérationnel de ces critères, nous les avons appliqués pour analyser l'activité

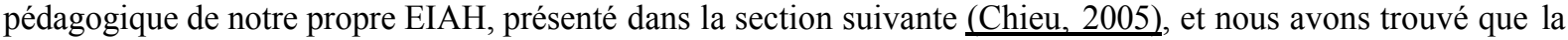
réalisation de l'analyse n'était ni difficile, ni laborieuse. Nous discuterons plus avant l'utilisation des critères de 
Jonnaert et Vander Borght dans la section qui suit.

\subsection{Critères proposés par Chieu, Frenay et Milgrom}

Milgrom et Frenay sont professeurs à l'Université catholique de Louvain. Milgrom effectue des recherches sur la pédagogie active dans le contexte des EIAH ; Frenay s'intéresse aux processus d'apprentissage et de transfert de connaissances et aux dispositifs pédagogiques dans l'enseignement universitaire. Chieu est docteur en sciences appliquées de l'Université catholique de Louvain; ses recherches portent sur l'informatique et les sciences cognitives et pédagogiques dans le contexte des EIAH. Ensemble, ils ont proposé une liste de critères pour caractériser non pas la totalité des facettes du constructivisme, mais uniquement certains aspects qu'ils jugent essentiels ; cette liste de critères peut être utilisée comme un cadre pour l'évaluation des conditions d'apprentissage dans une variété de situations d'enseignement et d'apprentissage.

\subsubsection{Approche pédagogique}

Notre approche pédagogique s'appuie explicitement sur le constructivisme cognitif. Dans ce paradigme, Driscoll (Driscoll, 2000) examine une variété de perspectives constructivistes et identifie cinq facettes majeures relatives à la création de conditions d'apprentissage : (a) le raisonnement, la pensée critique et la résolution de problèmes

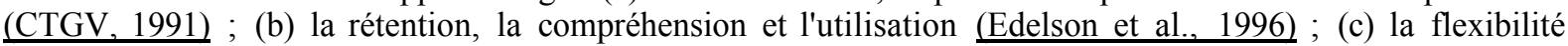
cognitive (Feltovich et al., 1996), (Spiro et al., 1991) ; (d) l'autorégulation (Duffy et Cunningham, 1996) et (e) la réflexion attentive et la flexibilité épistémologique (LDHG, 1992).

Parmi les cinq facettes du constructivisme, nous avons choisi de travailler en premier lieu sur des critères pour caractériser la flexibilité cognitive et ce, pour trois raisons principales. Premièrement, nous constatons que les principes pédagogiques de la flexibilité cognitive reflètent les caractéristiques de base du constructivisme (Spiro et al. 1991). Deuxièmement, nous voyons que la flexibilité cognitive est un point commun important à de nombreux chercheurs constructivistes (Bourgeois et Nizet, 1999), (Driscoll, 2000), (Spiro et Jehng, 1990). Et, troisièmement, nous pensons que les TIC sont particulièrement bien placées pour faciliter la création de conditions d'apprentissage soutenant la flexibilité cognitive, comme Driscoll (Driscoll, 2000) et Wilson (Wilson, 1996) l'ont montré dans quelques exemples de hypermédias.

La flexibilité cognitive est «la capacité d'une personne à structurer ou restructurer ses propres connaissances, de différentes façons, afin de répondre de manière adaptative à une variété de nouvelles situations " (Spiro et Jehng, 1990). Voici quelques exemples de flexibilité cognitive chez l'étudiant, d'autres exemples figurent dans $\underline{\text { (Spiro et Jehng, 1990) }}$ et dans (Chieu, 2005) :

- Lorsque l'étudiant est confronté à un nouveau problème, il essaie d'analyser systématiquement différents aspects du problème et d'utiliser différentes approches qu'il a appliquées avec succès dans le passé pour résoudre des problèmes plus ou moins similaires en vue de trouver une solution la plus complète possible.

- Lorsque l'étudiant est confronté à un nouveau concept, il essaie de réaliser différentes activités d'apprentissage dans différents contextes afin de rencontrer une variété d'aspects du nouveau concept.

- Lorsque l'étudiant discute avec ses pairs, il essaie de poser systématiquement des questions de nature à éliciter les points de vue de ses pairs.

Pour favoriser la flexibilité cognitive dans l'enseignement, surtout dans l'acquisition de connaissances avancées, Feltovich, Spiro et leurs collègues (Feltovich et al., 1996) ont présenté une théorie de la flexibilité cognitive dans laquelle ils ont argumenté en faveur de l'utilisation de multiples formes de modèles pédagogiques, de multiples métaphores et analogies et de multiples interprétations de la même information. Un autre point de vue au sujet de la flexibilité cognitive, proposé par Bourgeois et Nizet (Bourgeois et Nizet, 1999), souligne que l'enseignant devrait encourager l'étudiant à explorer le plus profondément possible de nouvelles connaissances, dans diverses situations concrètes. D'autre part, ils ont ajouté qu'il est nécessaire de donner des moyens permettant à l'apprenant d'analyser et d'évaluer ces nouvelles connaissances "de l'extérieur". Selon cette approche, l'enseignant est responsable de trois activités : (a) encourager les étudiants à exprimer leurs points de vue personnels, (b) organiser la confrontation des points de vue des étudiants, et (c) fournir les outils méthodologiques permettant aux étudiants de traiter ces différents points de vue.

Driscoll (Driscoll, 2000) a examiné les hypothèses suggérées par Spiro et ses collègues et identifié deux conditions d'apprentissage principales pour la flexibilité cognitive : (a) les multiples modes d'apprentissage, c'est-à-dire les multiples représentations de contenus d'apprentissage, les multiples façons et méthodes pour explorer des contenus d'apprentissage; et (b) les multiples perspectives sur l'apprentissage, c'est-à-dire l'expression, la confrontation et le traitement de multiples points de vue. Nous acceptons les deux conditions d'apprentissage proposées par Driscoll parce qu'elles semblent incarner différents points de vue suggérés par d'autres théoriciens en éducation.

\subsubsection{Critères}




\begin{tabular}{|c|c|c|}
\hline \multirow{2}{*}{$\begin{array}{l}\text { Composants } \\
\text { d'apprentissage }\end{array}$} & \multicolumn{2}{|c|}{ Conditions d'apprentissage } \\
\hline & Multiples modes d'apprentissage & Multiples perspectives sur l'apprentissage \\
\hline $\begin{array}{l}\text { Contenus } \\
\text { d'apprentissage }\end{array}$ & $\begin{array}{l}\text { MM1 : le même contenu d'apprentissage } \\
\text { présentant des concepts et leurs relations est } \\
\text { représenté en différentes formes telles que le } \\
\text { texte, les images, l'audio, la vidéo et les } \\
\text { simulations. }\end{array}$ & $\begin{array}{l}\text { MP1 : le même concept abstraire est expliqué, utilisé } \\
\text { et appliqué systématiquement avec d'autres concepts } \\
\text { dans une diversité d'exemples d'utilisation, exercices } \\
\text { et études de cas dans des situations appropriées, } \\
\text { "réalistes" et complexes. }\end{array}$ \\
\hline $\begin{array}{l}\text { Dispositifs } \\
\text { pédagogiques }\end{array}$ & $\begin{array}{l}\text { MM2 : l'apprenant est encouragé à étudier le } \\
\text { même concept abstraire pour différents } \\
\text { objectifs, en différents moments, par } \\
\text { différentes méthodes et activités telles que la } \\
\text { lecture, l'exploration, la discussion et la } \\
\text { réorganisation de connaissances. }\end{array}$ & $\begin{array}{l}\text { MP2 : lorsque l'apprenant est confronté à un nouveau } \\
\text { concept, il est encouragé à explorer le plus loin } \\
\text { possible les relations entre celui-ci avec d'autres } \\
\text { concepts dans des situations appropriées, "réalistes" } \\
\text { et complexes. } \\
\text { MP3 : lorsque l'apprenant est confronté à un nouveau } \\
\text { concept, il est encouragé à explorer différentes } \\
\text { interprétations de celui-ci (par d'autres auteurs et par } \\
\text { ses pairs), à exprimer son point de vue personnel sur } \\
\text { le nouveau concept et à donner des feedback sur les } \\
\text { points de vue d'autres personnes. } \\
\text { MP4 : lorsque l'apprenant est confronté à un nouveau } \\
\text { concept, il est encouragé à examiner, analyser et } \\
\text { synthétiser une diversité de points de vue sur le } \\
\text { nouveau concept. }\end{array}$ \\
\hline $\begin{array}{l}\text { Interactions } \\
\text { humaines }\end{array}$ & $\begin{array}{l}\text { MM3 : le nombre de participants, le type de } \\
\text { participant (l'apprenant, l'enseignant, } \\
\text { l'expert, etc.), les outils de communication } \\
\text { et les localisations (dans la classe, le } \\
\text { campus ou le monde, etc.) sont variés. }\end{array}$ & $\begin{array}{l}\text { MP5 : pendant la discussion, l'apprenant est } \\
\text { encouragé à diversifier - le plus loin possible - les } \\
\text { différents points de vue à propos du sujet discuté. }\end{array}$ \\
\hline $\begin{array}{l}\text { Dispositifs } \\
\text { d'évaluation }\end{array}$ & $\begin{array}{l}\text { MM4 : pendant le processus } \\
\text { d'apprentissage, l'apprenant est encouragé à } \\
\text { utiliser différents méthodes et outils } \\
\text { d'évaluation, en différents moments et en } \\
\text { différents contextes pour démontrer sa } \\
\text { capacité à résoudre différents problèmes. }\end{array}$ & $\begin{array}{l}\text { MP6 : pendant le processus de résolution d'un } \\
\text { problème, l'apprenant est encouragé à confronter de } \\
\text { multiples façons à résoudre le problème et de } \\
\text { multiples solutions possibles pour le problème. }\end{array}$ \\
\hline
\end{tabular}

Tableau 3 : Ensemble des critères opérationnels pour la flexibilité cognitive

Nous définissons un critère opérationnel pour la flexibilité cognitive comme un test qui permet de répondre aisément à la question de savoir si une condition d'apprentissage reflète les principes pédagogiques de la flexibilité cognitive. Pour proposer un ensemble complet de critères opérationnels, nous avons d'abord considéré les deux conditions d'apprentissage identifiées précédemment. Puis, à partir de nombreuses implications pédagogiques proposées par des chercheurs en sciences de l'éducation (voir la section 1.1), nous avons identifié quatre composants d'apprentissage importants : (a) les contenus d'apprentissage (les exemples, exercices, études de cas, etc.) ; (b) les dispositifs pédagogiques (les méthodes et outils fournis aux apprenants pour explorer des contenus d'apprentissage) ; (c) les interactions humaines (les moyens et techniques pour engager l'enseignant et les apprenants dans des discussions) ; et (d) les dispositifs d'évaluation (les problèmes et outils pour déterminer si l'apprenant atteint ses objectifs d'apprentissage). Finalement, pour chacune des deux conditions d'apprentissage et pour chacun des quatre composants d'apprentissage, nous avons proposé un ou plusieurs critères pour vérifier la présence de la condition d'apprentissage dans le composant d'apprentissage. Nous avons distingué les quatre composants d'apprentissage en vue de faciliter la proposition et l'application de nos critères. Le Tableau 3 présente l'ensemble de ces critères opérationnels $(\mathrm{MM}=$ Multiples Modes, MP = Multiples Perspectives). La section 3.3.3 explique la pertinence de ces critères.

\subsubsection{Application}

Nous utilisons l'ensemble des critères opérationnels comme un guide (Chieu, 2005) afin de : (a) proposer un processus opérationnel pour créer des conditions d'apprentissage favorisant la flexibilité cognitive, (b) développer une plate-forme de e-Learning basée sur le Web soutenant la flexibilité cognitive (COFALE : Cognitive Flexibility in Ádaptive Learning Environments), et (c) évaluer des conditions d'apprentissage favorisant la 
flexibilité cognitive (par exemple un cours créé en utilisant COFALE). Dans cette section-ci, nous montrons comment nous avons évalué un cours géré par COFALE comme un exemple d'utilisation de nos critères.

Nous avons utilisé COFALE pour concevoir un cours sur le concept, fondamental en informatique, de récursion (Henderson et Romero, 1989). De nombreux enseignants considèrent que l'enseignement et l'apprentissage du concept de récursion sont difficiles (Anderson et al., 1988), car le concept est complexe et peu fréquent dans la vie quotidienne. Pour la discussion qui suit, nous supposons un apprenant "novice" (Bob), qui maîtrise la programmation "traditionnelle" (dans notre cas : le langage Java) avec le concept de l'itération. Bob utilise COFALE pour apprendre le concept de récursion (c'est-à-dire développer la capacité à utiliser la récursion pour résoudre des problèmes). Nous supposons aussi qu'un tuteur et un certain nombre d'autres apprenants (les pairs) participent à la même expérience d'apprentissage. Par la suite, pour chacun de nos critères relatifs à la flexibilité cognitive, nous montrons comment COFALE fournit à Bob des situations d'apprentissage satisfaisant le critère.

Bob voudrait développer sa capacité à implémenter des solutions récursives pour une variété de problèmes. Il navigue grâce au menu local ("Local Menu") et lit la définition et les exemples des concepts importants : récursion, algorithmes et méthodes récursifs (Figure 1 : Zone 1). Puis, Bob est encouragé à explorer une situation à propos des expressions arithmétiques (Figure 1 : Zone 2).

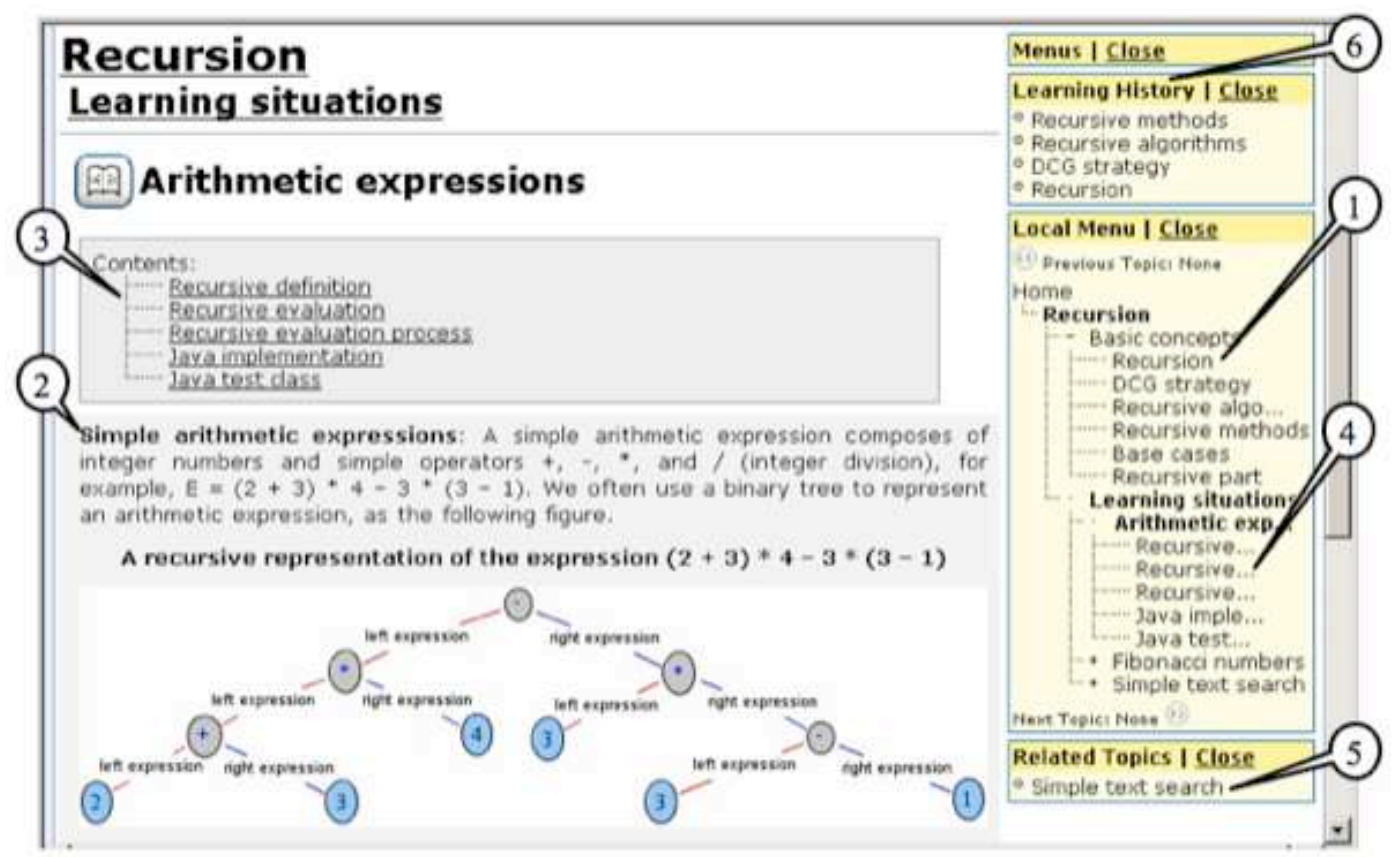

Figure 1 : Une partie de l'hyperespace d'apprentissage de Bob

Critère MM1 : Dans la situation des expressions arithmétiques, le concepteur du cours induit Bob à examiner les multiples représentations du concept de récursion en utilisant les hyperliens présentés dans la Zone 3 ou la Zone 4 de la Figure 1 : une définition textuelle, deux simulations et une implémentation en Java.

Critère MP1 : Après avoir exploré la première situation (les expressions arithmétiques), Bob est encouragé à explorer la deuxième situation à propos de la recherche simple de textes en cliquant sur un hyperlien dans le menu "Related Topics" (Figure 1, Zone 5). Dans cette situation, Bob voit comment appliquer la récursion pour représenter un texte (une liste de mots) comme une liste chaînée et rechercher une phrase dans un document. Ici, le concepteur du cours a préparé une diversité de situations pour aider Bob à comprendre comment appliquer le concept de récursion dans différents contextes : les expressions arithmétiques illustrent, de manière naturelle, l'utilisation de la récursion dans les arbres binaires ; la recherche simple de textes explique l'utilisation de la récursion dans les listes chaînées.

Critère MP2 : Pendant que Bob explore la situation de la recherche simple de textes, COFALE lui présente un hyperlien (dans le menu "Related Topics") qui l'encourage à examiner le concept de listes chaînées, qui est en relation avec la récursion, car les listes chaînées sont un type particulier de structures de données récursives. Similairement, pendant qu'il examine le concept de listes chaînées, Bob peut retourner à l'hyperespace de la récursion en cliquant sur un des hyperliens présentés dans les menus "Related Topics" et "Learning History" (Figure 1, Zone 6). Ce dernier menu contient les hyperliens vers les pages Web que Bob a visitées récemment. Les deux menus aident Bob également à naviguer intelligemment pour éviter de se perdre dans l'hyperespace d'apprentissage.

Critère MM2 : À la fin de chaque page Web, COFALE présente à Bob des activités d'apprentissage pour le 
guider et l'amener à explorer l'hyperespace d'apprentissage. Par exemple, après avoir exploré la situation des expressions 'contextes pour étudier plus à fond le concept de récursion.

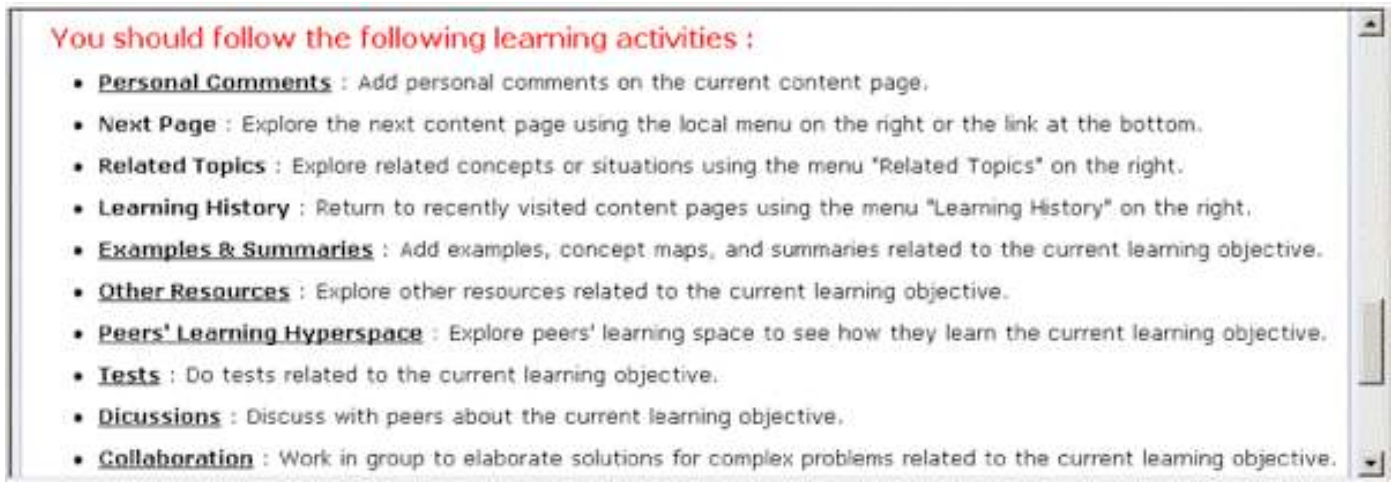

Figure 2 : Activités d'apprentissage proposées à Bob

Critère MP3 : Pour satisfaire ce critère, COFALE propose à Bob les quatre activités d'apprentissage suivantes :

a. Ajouter des commentaires sur les contenus d'apprentissage créés par le concepteur du cours, par exemple : reformuler les points principaux de la définition du concept de récursion dans ses propres termes (Figure 2 : "Personal Comments").

b. Ajouter ses propres exemples, par exemple : un phénomène récursif dans sa vie quotidienne (Figure 2 : "Examples \& Summaries").

c. Explorer des ressources externes, par exemple : les cours en ligne de Java (Eck, 2004), (Kjell, 2003) dans lesquels les auteurs illustrent un nombre important d'exemples récursifs (Figure 2 : "Other Resources").

d. Explorer des hyperespaces d'apprentissage de ses pairs, par exemple se loguer dans un espace d'apprentissage d'un apprenant "expert" afin d'examiner ses exemples récursifs à lui (Figure 2 : "Peers' Learning Hyperspace").

Critère MP4 : COFALE suggère à Bob de produire des résumés sur les points de vue d'autres sources et de ses pairs (Figure 2 : "Examples \& Summaries"). Par exemple, COFALE fournit à Bob un tableau vide lui permettant de confronter ses propres définitions du concept de récursion, de méthodes récursives et de la résolution de problèmes avec celles de ses pairs.

Critère MM3 : COFALE encourage Bob à travailler avec ses pairs (Figure 2 : "Discussions"), parfois avec la participation du tuteur, en utilisant de multiples outils de communication tels que les courriels, les forums et les salles de conversation (chat rooms). COFALE incite aussi Bob à utiliser un site Web (Java World, 2006) pour poser aux experts des questions à propos de la récursion.

Critère MP5 : Pour satisfaire ce critère, COFALE attache, à chacun des outils de discussion, une liste de questions générales et une liste de questions spécifiques en rapport avec la récursion. Ces listes de questions permettent à Bob de solliciter les points de vue de ses pairs. Ces questions incluent "Pourquoi ?", "Quelle est la source de ton information ?", "Y a-t-il d'autres explications pour cette question ?", "Pourquoi la récursion est-elle utile dans ce problème ?", "Comment arrives-tu de l'énoncé du problème à ta solution récursive ?".

Critère MM4 : A différents moments, par exemple après l'exploration de multiples situations d'apprentissage, Bob est amené à effectuer deux activités d'évaluation :

a. Tests individuels : Bob est confronté à une situation d'évaluation dans laquelle le concepteur du cours lui demande de faire un certain nombre de tests tels que calculer toutes les combinaisons des pas de 1 mètre, 2 mètres ou 4 mètres que peut utiliser un robot pour effectuer un trajet de $n$ mètres où $n$ est un nombre entier positif.

b. Travail en groupe : COFALE amène Bob à participer à un groupe de deux ou trois étudiants pour résoudre des problèmes non triviaux dans un système de fichiers à structure arborescente ; il s'agit, par exemple, de lister de manière récursive tous les fichiers et les sous-répertoires dans un répertoire donné.

Critère MP6 : Dans la situation du robot, Bob est encouragé à appliquer et comparer la méthode itérative et la méthode récursive. Dans la situation de la gestion de fichiers, le tuteur amène Bob et ses coéquipiers à confronter et à comparer différentes solutions possibles : (a) lister d'abord les fichiers et sous-répertoires dans le répertoire donné, puis dans ses sous-répertoires et (b) lister d'abord les fichiers et sous-répertoires dans les sous-répertoires du répertoire donné, puis dans le répertoire donné lui-même.

L'analyse précédente montre que le cours sur la récursion satisfait tous les critères proposés plus haut pour la flexibilité cognitive. 


\subsubsection{Discussion}

Bien que notre approche se centre sur les principes pédagogiques de la flexibilité cognitive, une seule des facettes du constructivisme cognitif, nous n'avons pas ignoré la dimension de l'interaction sociale (voir les critères MM3 et MP5, par exemple). En effet, une analyse du cours de récursion sous COFALE effectuée en utilisant les critères proposés par Jonnaert et Vander Borght (voir la section 3.2) montre que l'activité pédagogique présentée satisfait tous leurs critères (Chieu, 2005). Nous pouvons donc affirmer que notre modèle pédagogique est cohérent avec l'approche socioconstructiviste et interactive de Jonnaert et Vander Borght.

Nous sommes convaincus qu'il est possible d'exploiter d'autres facettes du constructivisme que la flexibilité cognitive (par exemple : la résolution de problèmes) de manière similaire afin de proposer des critères pour ces autres facettes. Cependant, à notre avis, transformer des principes pédagogiques de la résolution de problèmes en critères opérationnels sera difficile, car la résolution de problèmes dépend du sujet à apprendre (Weber et Brusilovsky, 2001) : on ne résout pas les problèmes de manière similaire en mathématiques, en ingénierie ou en médecine.

En plus de notre analyse du cours de récursion, nous avons aussi utilisé l'ensemble de nos critères pour évaluer un certain nombre de EIAH existants (Chieu et al., 2006). L'analyse montre qu'il y a de nombreuses manières d'exploiter les TIC afin de satisfaire ces critères. La réalisation d'une telle évaluation n'est pas très coûteuse (1 personne-heure en moyenne pour chaque EIAH). Nous pouvons donc affirmer que nos critères pour la flexibilité cognitive sont effectivement opérationnels.

Cependant, après avoir validé ces critères (voir la section 3.4), nous devons encore formuler plusieurs remarques importantes pour l'application de ces critères. Voir aussi les discussions de (Jonnaert et Vander Borght, 2003) à propos de l'utilisation de leurs critères :

- La qualité de la satisfaction des critères est importante : l'ensemble des critères est là pour aider les concepteurs, chercheurs et praticiens à créer et évaluer des conditions d'apprentissage. Cependant, leur expertise dans le sujet enseigné est essentielle. Par exemple, pour satisfaire le critère MP1, le concepteur du cours doit être un expert en récursion pour pouvoir préparer une diversité de situations d'apprentissage significatives pour l'apprenant : préparer une diversité de situations qui expliquent différents aspects ou interprétations d'un nouveau concept (qualité) serait plus important que préparer beaucoup de situations qui n'expliquent qu'un ou deux aspects du nouveau concept (quantité).

- Il n'est sans doute pas nécessaire de satisfaire tous les critères : à l'heure actuelle, il n'existe aucune preuve qui montre que satisfaire tous les critères que nous proposons est toujours préférable à satisfaire une partie de ces critères. Il est possible que la satisfaction de quelques critères suffit pour les cours introductifs et que satisfaire la plupart des critères ou tous les critères est nécessaire pour les cours avancés (Chieu et al., 2006). Par exemple, le cours sur les concepts de vitesse, accélération, distance, etc. en physique, qui a été conçu dans le cadre du projet SimQuest (de Jong et al., 2004), ne satisfait qu'une moitié de nos critères pour la flexibilité cognitive, mais il a aidé néanmoins les élèves à atteindre leurs objectifs d'apprentissage de manière effective.

- Parmi les critères, certains sont plus importants que d'autres : par exemple, le critère MP1 est plus important que le critère MM1. En effet, les multiples représentations de contenus d'apprentissage (MM1) facilitent la perception de l'apprenant à propos d'un aspect d'un nouveau concept, mais c'est la diversité des situations d'apprentissage (MP1) qui suscite le conflit cognitif salutaire chez l'apprenant. En réalité, satisfaire le critère MP1 est plus difficile que satisfaire le critère MM1. Par exemple, nous avons trouvé qu'à la fin des chapitres de nombreux livres de référence, les auteurs présentent souvent des séries d'exercices, mais ces derniers n'illustrent que peu d'interprétations différentes d'un nouveau concept complexe (Chieu, 2005).

- Les critères ne sont pas totalement indépendants les uns des autres : le concepteur de cours doit être conscient de cette caractéristique pour appliquer effectivement l'ensemble des critères. Par exemple, les critères MP3 et MP4 renforcent le critère MM2, car ils encouragent l'apprenant à effectuer une variété d'activités d'apprentissage.

- L'ensemble des critères proposés n'est pas définitif : on peut certainement le modifier. Par exemple nous ne doutons pas qu'il soit possible d'organiser un critère en sous-critères ou de rassembler plusieurs critères en un seul critère, ou même de refuser une partie de ces critères et d'en proposer de nouveaux, selon l'interprétation personnelle que chacun voudra donner à la flexibilité cognitive.

\subsection{Validation des trois approches}

Bien que TACTICS ait été utilisé pour promouvoir l'apprentissage collaboratif au sein d'une communauté d'élèves, nous n'avons pas trouvé d'indications relatives à l'existence d'un volet expérimental qui aurait permis de démontrer la cohérence entre les trois critères proposés par les auteurs et les conditions d'apprentissage fournies par TACTICS. Il n'est donc pas possible de répondre à une question telle que "Après un apprentissage avec l'aide de TACTICS, est-ce que chaque élève construit véritablement ses propres connaissances par lui-même plutôt que par transmission ?" 
Les auteurs de la deuxième approche ne mentionnent pas l'existence d'une expérimentation effectuée pour valider l'ensemble des critères du modèle socioconstructiviste et interactif qu'ils proposent. Ce n'est pas surprenant, parce que l'objectif des auteurs, en proposant leurs critères, était essentiellement de donner une définition la plus complète possible du concept d'apprentissage en s'appuyant sur leur modèle socioconstructiviste et interactif.

Quant à nous, nous avons réalisé une évaluation préliminaire - et de portée limitée - de notre approche par le cours que nous avons réalisé sur la récursion. L'objectif principal de l'étude était d'examiner des résultats d'apprentissage avec l'aide de COFALE, en particulier la flexibilité cognitive chez l'apprenant. Neuf étudiants de première année en informatique à l'Université catholique de Louvain, avec certaines connaissances préalables de la programmation en Java mais aucune connaissance de la récursion, ont été répartis aléatoirement en deux groupes : quatre dans le groupe de COFALE et cinq dans le groupe de contrôle. Nous avons organisé l'étude en quatre phases : pré-test, expérimentation, post-test, et entretien. Les deux groupes ont été soumis aux mêmes pré-test, post-test et questions lors de l'entretien. Pour la phase expérimentale, ils ont reçu le même cours magistral (pendant 45 minutes) et les mêmes consignes (pendant 2 heures). Seule différence : après le cours magistral, le groupe de COFALE a exploré le cours sous COFALE et le groupe de contrôle a exploré librement un chapitre d'un livre de référence (Lewis et Loftus, 2003) qui traite du sujet de la récursion (pendant 1 heure).

Un certain nombre de résultats encourageants pour l'apprentissage avec l'aide de COFALE ont pu être dégagés de cette première expérience. Les étudiants dans les deux groupes ont maîtrisé la récursion à un niveau appréciable. Cependant, le comportement d'apprentissage du groupe de COFALE semble plus cohérent avec la flexibilité cognitive que celui du groupe de contrôle. Par exemple : dans le post-test, les membres du groupe de COFALE ont fait plus attention à activer leurs connaissances préalables de différentes manières, à analyser de différents aspects d'un nouveau problème et à proposer une solution la plus complète possible que le groupe de contrôle; dans l'entretien, le groupe de COFALE a défini le concept de récursion plus clairement et plus correctement que le groupe de contrôle. De plus, le commentaire suivant d'un étudiant du groupe COFALE peut montrer l'effectivité des critères MM1 et MP1 (l'indication est en italique) :

"COFALE est bon, personnalisé... Nous pouvons travailler n'importe où, soumettre des exercices en ligne... Il n'y a pas beaucoup dans une page Web... Beaucoup d'exemples, ils sont clairs et bien expliqués, dans chaque exemple on ne donne pas la solution immédiatement, il y a une page pour expliquer comment réfléchir, une page pour expliquer comment arriver à la solution et une page pour présenter la solution. Ceci aide à construire notre propre solution par nous-mêmes."

Dans la section suivante, nous tirons des conclusions de l'analyse des trois approches présentées.

\section{Conclusion}

Le Tableau 4 résume notre analyse de trois approches basées sur des critères.

\begin{tabular}{|c|c|c|c|}
\hline Auteurs & $\begin{array}{l}\text { Brousseau, } \\
\text { Vázquez-Abad }\end{array}$ & $\begin{array}{l}\text { Jonnaert, Vander } \\
\text { Borght }\end{array}$ & $\begin{array}{l}\text { Chieu, } \\
\text { Milgrom, } \\
\text { Frenay }\end{array}$ \\
\hline $\begin{array}{l}\text { Approche } \\
\text { pédagogique }\end{array}$ & $\begin{array}{l}\text { Constructivisme } \\
\text { cognitif, } \\
\text { socioconstructivisme }\end{array}$ & $\begin{array}{l}\text { Approche } \\
\text { socioconstructiviste } \\
\text { et interactive }\end{array}$ & $\begin{array}{l}\text { Flexibilité } \\
\text { cognitive }\end{array}$ \\
\hline Critères & $\begin{array}{l}3 \\
\text { générales }\end{array}$ & $\begin{array}{l}11 \quad \text { critères } \\
\text { opérationnels }\end{array}$ & $\begin{array}{l}10 \quad \text { critères } \\
\text { opérationnels }\end{array}$ \\
\hline Application & $\begin{array}{l}\text { Analyse d'une } \\
\text { activité pédagogique } \\
\text { de TACTICS }\end{array}$ & $\begin{array}{lr}\text { Analyse } & \text { des } \\
\text { définitions } & \text { du } \\
\text { concept } & \text { de } \\
\text { l'apprentissage } & \end{array}$ & $\begin{array}{l}\text { Analyse des } \\
\text { conditions } \\
\text { d'apprentissage } \\
\text { des EIAH }\end{array}$ \\
\hline Validation & $\begin{array}{l}\text { Pas } \\
\text { d'expérimentation } \\
\text { spécifique pour } \\
\text { valider les critères }\end{array}$ & $\begin{array}{l}\text { Pas } \\
\text { d'expérimentation } \\
\text { spécifique pour } \\
\text { valider les critères }\end{array}$ & $\begin{array}{l}\text { Evaluation } \\
\text { préliminaire } \\
\text { du cours sur la } \\
\text { récursion }\end{array}$ \\
\hline
\end{tabular}

Tableau 4 : Résumé de l'analyse des trois approches

Les conclusions suivantes peuvent être tirées de notre analyse : 
- Il existe de multiples paradigmes du constructivisme: Bien que les auteurs des trois approches s'appuient sur les mêmes principes de base du constructivisme, ils les ont interprétés différemment dans la pratique. En effet, nous nous focalisons sur le développement individuel, tandis que les autres auteurs mettent l'accent sur la combinaison entre le développement individuel et la négociation sociale. Cependant, les auteurs des trois approches ont un point commun : clarifier les principes pédagogiques en proposant des critères, qui ont pour but de faciliter l'évaluation et/ou la création de conditions d'apprentissage favorisant l'approche constructiviste.

- Vers des critères opérationnels pour l'évaluation des conditions d'apprentissage : Alors qu'il est malheureusement pratique courante d'affirmer qu'une condition d'apprentissage proposée favorise le constructivisme, sans toutefois offrir la moindre forme de justification ou d'argumentation, les critères opérationnels peuvent être une grille d'analyse efficace pour ce type d'affirmation. Nous soulignons le qualificatif "opérationnel" puisque, d'après nous, des critères tels que les indications générales de Brousseau et Vázquez-Abad ne suffiraient pas pour réaliser des analyses pertinentes et précises. Nous pensons que les critères opérationnels tels que ceux proposés par Jonneart et Vander Borght ou les nôtres fournissent un moyen facile et prêt à utiliser pour alléger sensiblement un travail d'évaluation du constructivisme effectif.

Les critères opérationnels présentés dans le présent article peuvent être réutilisables dans une variété de situations d'enseignement, tant pour la formation traditionnelle (Jonnaert et Vander Borght, 2003) que pour les EIAH (Chieu et al., 2006). Cependant, comme la discussion de la section 3.3.4 l'indique, ces critères ne sont ni définitifs, ni normatifs, ni prescriptifs. Chaque praticien doit trouver sa propre manière de les appliquer dans sa situation d'enseignement, de les modifier en cas de besoin (par exemple, pour mieux s'adapter à une situation particulière) ou de les rejeter et de proposer de nouveaux critères (par exemple, pour son propre modèle pédagogique). L'unique contrainte que les chercheurs, concepteurs et praticiens devraient s'imposer dans tous les cas, c'est la cohérence entre l'ensemble des critères qu'ils proposent et l'approche pédagogique qu'ils poursuivent (Jonnaert et Vander Borght, 2003). De plus, il faut noter que les auteurs de trois approches ont tenu à montrer comment ils en sont arrivés à proposer leurs critères; ceci est important parce que cela peut aider les chercheurs, concepteurs et praticiens dans la création de leurs propres critères. A titre d'exemple, afin de proposer des critères opérationnels pour d'autres facettes du constructivisme que la flexibilité cognitive, par exemple la résolution de problèmes, on pourrait commencer par identifier des conditions d'apprentissage favorisant la résolution de problèmes, puis proposer des critères pour chacune de ces conditions d'apprentissage et pour chacun des quatre composants d'apprentissage présentés dans la section 3.3.2 (c'est-à-dire, les contenus d'apprentissage, les dispositifs pédagogiques, les interactions humaines et les dispositifs d'évaluation).

- Vers un processus opérationnel pour la création de conditions d'apprentissage adéquates : Une tâche importante, différente de l'évaluation des conditions d'apprentissage, est la création de conditions d'apprentissage conformes à un modèle donné au sein du processus de construction d'un environnement d'apprentissage (Jonnaert et Vander Borght, 2003); (Chieu, 2005). La question principale est ici de déterminer comment concevoir un environnement d'apprentissage soutenant les principes pédagogiques que l'on a choisis ou, plus spécifiquement, satisfaisant un ensemble (ou sous-ensemble) de critères. Après avoir proposé des critères pour leur modèle SCI, Jonneart et Vander Borght ont aussi fourni un processus pour aider les praticiens à créer des conditions d'apprentissage en cohérence avec leur modèle SCI. Ce processus précise les étapes à effectuer par les concepteurs dans les trois phases principales d'un enseignement : pré-active, interactive et post-active (pour plus de détails, voir Jonnaert et Vander Borght, 2003, chapitre 6). De même, sur la base de nos critères, nous avons proposé un processus opérationnel pour aider les concepteurs à créer des environnements d'apprentissage soutenant la flexibilité cognitive. Ce processus se compose d'activités de conception organisées dans les trois phases pré-active, interactive et post-active. Chaque activité se compose d'une ou plusieurs opérations que les concepteurs peuvent effectuer afin de créer ou d'évaluer des conditions d'apprentissage pour les étudiants. Notre processus de conception satisfait tous les critères pour la flexibilité cognitive (Chieu, 2005, chapitre 3).

Comme les critères, les processus de conception peuvent être réutilisés afin de créer des environnements d'apprentissage dans une variété de contextes d'apprentissage. Une fois encore, les processus de conception ne sont pas définitifs : les concepteurs doivent évidemment les adapter à leurs propres situations d'enseignement.

- Vers une approche opérationnelle pour la conception des EIAH : Nous considérons l'ensemble des critères et le processus de conception décrits ci-dessus comme un cadre utile pour guider les concepteurs et les constructeurs de plates-formes d'apprentissage basées sur les TIC (voir COFALE dans la section 3.3). COFALE comporte non seulement des outils pour l'apprenant (Chieu et Milgrom, 2005), mais aussi des outils pour l'enseignant (Chieu, 2006). En d'autres mots, les concepteurs peuvent utiliser l'ensemble des critères et le processus de conception comme un modèle pédagogique et la plate-forme COFALE comme un moyen technologique afin de créer des EIAH soutenant explicitement la flexibilité cognitive. Un point important est que la conception et l'implémentation de COFALE au niveau informatique sont orientées vers l'ensemble de nos critères : chaque outil d'apprentissage et chaque outil auteur dans COFALE doivent avoir une raison d'être, c'est-à-dire être présents pour satisfaire un ou plusieurs critères pour la flexibilité cognitive. Grâce à notre approche opérationnelle, la charge pour le développement de COFALE (6 personnes-mois) et pour la conception 
et la réalisation du cours sur la récursion (8 personnes-heures) peut être considérée comme relativement légère. Cela nous permet d'affirmer que l'approche opérationnelle est effective.

Un certain nombre de chercheurs dans le domaine des EIAH s'appuient sur nos conclusions. Basque, Dao et Contamines (Basque et al., 2005), par exemple, ont transformé des principes pédagogiques de l'apprentissage "situé" (situated learning) en critères opérationnels pour faciliter la conception et l'évaluation de leur EIAH, nommé CSV (Colloque Scientifique Virtuel). Balacheff (1995) a proposé un modèle "opérationnel", nommé $\mathrm{cK} \phi$, pour clarifier le processus cognitif de la résolution de problèmes chez l'apprenant. Luengo, Mufti-Alchawafa et Vadcard (Luengo et al., 2004), (Vadcard et Luengo, 2005), à partir du modèle cKф, ont appliqué une approche opérationnelle pour la conception d'un EIAH dans un domaine complexe, la chirurgie orthopédique.

- Vers des critères opérationnels pour l'expérimentation des EIAH : Après la mise à disposition d'un EIAH, un autre exercice que nous avons abordé dans la section 3.4 est sa validation avec des apprenants en situation, en particulier l'évaluation du comportement cognitif de ces apprenants. Cette évaluation est importante parce qu'elle permet aux auteurs d'un EIAH de savoir si leur environnement favorise véritablement l'apprentissage chez l'étudiant, selon les principes pédagogiques qu'ils ont appliqués (Chieu, 2005), (Wilson, 1997). Cet exercice est ardu, car il est évidemment impossible de savoir ce qui se passe exactement dans la "tête" de l'apprenant lorsqu'il apprend (Wilson, 1997). Par exemple, lors de l'évaluation préliminaire de COFALE, il nous a été difficile de trouver des questions pertinentes afin d'aider les apprenants à exprimer ce qui se passe en eux pendant leur processus d'apprentissage. Pour ce type d'exercice, nous pensons que les critères opérationnels sont très utiles, comme ceux pour l'évaluation des conditions d'apprentissage. Cependant, proposer des critères pour l'évaluation du comportement cognitif chez l'apprenant nous semble beaucoup plus difficile que proposer des critères pour l'évaluation des conditions d'apprentissage, parce que ces dernières sont observables tandis que le premier ne l'est pas. Nous espérons que les chercheurs en sciences de l'éducation et en cognition contribueront à résoudre ce type de problème dans le futur.

\section{Remerciements}

Nous voudrions remercier les membres du comité de lecture pour leurs commentaires pertinents et constructifs pour améliorer la qualité scientifique du présent texte. Nous remercions aussi Lucile Vadcard et Vanda Luengo (CLIPS-IMAG, Grenoble) pour avoir lu attentivement et commenté une version précédente du présent article.

\section{BIBLIOGRAPHIE}

ANDERSON J.R., PIROLLI, P., FARRELL, R. (1988). Learning to program recursive functions, In M. Chi, R. Glaser, \& M. Farr (Eds.), The nature of expertise (pp. 153-184), Hillsdale, NJ: Erlbaum.

ASTOLFI J-P. (1989). La didactique des sciences, Collection Que sais-je? Paris : Presses universitaires de France.

BALACHEFF N. (1995). Conception, Connaissance et Concept. In: Denise Grenier (ed.) Séminaire Didactique et Technologies Cognitives en Mathématiques (pp. 219-244). Grenoble, France.

BASQUE J., DAO K., CONTAMINE J. (2005). L'apprentissage « situé » dans les cours en ligne : le cas du Colloque Scientifique Virtuel (CSV). EIAH 2005. Montpellier, France (25-27 mai).

BOURGEOIS E., FRENAY M. (2002). Les modèles théoriques de l'apprentissage: Note de synthèse, Louvain-la-Neuve, BE : Cours EDFO 2106 Psychologie de l'apprentissage.

BOURGEOIS E., NIZET J. (1999). Apprentissage et formation des adultes, Paris, FR : Presses Universitaires de France, 1999.

BROUSSEAU N., VAZQUEZ-ABAD J. (2003). Analyse de la nature constructiviste d'une activité d'apprentissage collaboratif médié par les TIC, Canadian Journal of Learning and Technology, Vol. 29 n³.

BRUNER J.S. (1973). Going beyond the information given, New York: Norton, 1973.

BRUNER J.S. (1986). Actual minds, possible worlds, Cambridge, MA: Harvard University Press, 1986.

BRUNER J.S. (1996). The culture of education, Cambridge, MA: Harvard University Press, 1996.

CHIEU V.M., MILGROM E., FRENAY M. (2004). Constructivist learning: Operational criteria for cognitive flexibility. The $4^{\text {th }}$ IEEE International Conference on Advanced Learning Technology. Joenssu, Finland (August 30-September 1), pp. 221-225.

CHIEU V.M., MILGROM E. (2005). COFALE: An adaptive learning environment supporting cognitive flexibility. The $12^{\text {th }}$ International Conference on Artificial Intelligence in Education. Amsterdam, The Netherlands (18-22 July), pp. 491-498.

CHIEU V.M. (2005). Constructivist learning: An operational approach for designing adaptive learning 
environments supporting cognitive flexibility, Thèse de doctorat, Université catholique de Louvain, 2005.

CHIEU V.M., ANH D.T.V., HUNG P.K. (2006). An operational approach for analyzing ICT-based constructivist and adaptive learning systems. The $4^{\text {th }}$ IEEE International Conference on Computer Sciences: Research, Innovation \& Vision for the Future. Ho Chi Minh, Vietnam (12-16 February), pp. 177-186.

CHIEU V.M. (2006). COFALE: An authoring system for supporting cognitive flexibility. The $6^{\text {th }}$ IEEE International Conference on Advanced Learning Technology. Kerkrade, The Netherlands (July 5-July 7), pp. 335-339.

Cognition and Technology Group at Vanderbilt (1991). Technology and the design of generative learning environments, Educational Technology, Vol. 31 (May), pp. 34-40.

DE JONG T., VAN JOOLINGEN W., VAN DER MEIJ J. (2004). SimQuest discovery learning. Retrieved November 8 , 2004 from: http://www.simquest.nl.

DOISE W., MUGNY G. (1997). Psychologie sociale et développement cognitif, Paris, FR : Armand Colin, 1997.

DRISCOLL M.P. (2000). Psychology of learning for instruction, Massachusetts: Allyn and Bacon, 2000.

DUFFY T.M., CUNNINGHAM D.J. (1996). Constructivism: Implications for the design and delivery of instruction, In D.H. Jonassen (Ed.), Handbook of research for educational communications and technology (pp. 170-198), New York: Macmillan.

ECK D.J. (2004). Introduction to programming using Java, Retrieved January 27, 2004 from: http://oopweb.com/Java/Documents/IntroToProgrammingUsingJava/VolumeFrames.html.

EDELSON D.C., PEA R.D., GOMEZ L. (1996). Constructivism in the collaboratory, In B.G. Wilson (Ed.), Constructivist learning environments: Case studies in instructional design (pp. 151-164), Englewood Cliffs, NJ: Educational Technology Publications.

ERNEST P.(1991). The philosophy of mathematics education, Hamshire, UK: The Falmer Press, 1991.

FELTOVICH P.J., SPIRO R.J., COULSON R.L., FELTOVICH J. (1996). Collaboration within and among minds: Mastering complexity, individually, and in groups, In T. Koschmann (Ed.), CSCL: Theory and practice of an emerging paradigm (pp. 25-44), Mahweh, NJ: Erlbaum.

HENDERSON P.B., ROMERO F.J. (1989). Teaching recursion as a problem-solving tool using Standard ML. The ACM 20th SIGCSE Technical Symposium on Computer Science Education. Louisville, Kentucky.

Java World (2006). Java $Q \& A$, Consulté le 31 août 2006: http://www.javaworld.com/columns/jw-qna-index.shtml .

JONASSEN D.H., ROHRER-MURPHY L. (1999). Activity theory as a framework for designing constructivist learning environments, Educational Technology, Research \& Development, Vol. 47 n ${ }^{\circ}$, pp. 61-79.

JONASSEN D.H. (1999). Designing constructivist learning environments, In C.M. Reigeluth, Instructional design theories and models: Their current state of the art (pp. 215-239). Mahwah, NJ: Lawrence Erlbaum Associates.

JONNAERT P., VANDER BORGHT C. (2003). Créer des conditions d'apprentissage : Un cadre de référence socioconstructiviste pour une formation didactique des enseignants, Bruxelles, BE : De Boeck-Université, Perspectives en Éducation et Formation, 2003.

JONNAERT P. (1995). Entrer dans l'apprentissage scolaire. In G. Forges, (éd.), Enfants issus de l'immigration et apprentissage du français langue seconde, pp. 15-53. Paris, FR : Didier-Erudition.

KINSHUK, LOOI C.K., SUTINEN E., SAMPSON D., AEDO I., UDEN L., KÄHKÖNEN E. (2004). Proceedings of the 4th IEEE International Conference on Advanced Learning Technologies, IEEE Computer Society, 2004.

KJELL B. (2003). Introduction to computer science using Java, Retrieved November 8, 2004 from: http://chortle.ccsu.edu/CS151/cs151java.html.

Language Development and Hypermedia Group (1992). Bubble dialogue: A new tool for instruction and assessment, Educational Technology, Research \& Development, Vol. 40 n², pp. 59-67.

LEWIS J., LOFTUS W. (2003). Java software solutions. Boston: Addison Wesley Longman.

LUENGO V., MUFTI-ALCHAWAFA D., VADCARD L. (2004). The knowledge like the object of interaction in an orthopaedic surgery-learning environment. The $7^{\text {th }}$ International Conference on ITS. Maceió, Alagoas, Brazil (August 30-September 3).

PIAGET J. (1975). L'équilibration des structures cognitives, Paris, FR: PUF, 1975.

REEVES T.C., OKEY J.R. (1996). Alternative assessment for constructivist learning environments, In B.G. Wilson (Ed.), Constructivist learning environments: Case studies in instructional design (pp. 191-202), Englewood Cliffs, NJ: Educational Technology Publications.

ROGOFF B. (1998). Cognition as a collaborative process, In W. Damon, D. Kuhn, \& R.S. Siegler (Eds.), Handbook of child psychology (5 ${ }^{\text {th }}$ ed., Vol. 2), New York: Wiley. 
SALOMON G. (1994). Interaction of media, cognition, and learning, Mahwah, NJ: Lawrence Erlbaum Associates, 1994.

SANTROCK J.W. (2001). Educational psychology, New York: McGraw-Hill, 2001.

SHEPARD L.A. (1991). Psychometricians' beliefs about learning, Educational Researcher, pp. 2-16.

SLAVIN R. E. (1995). Cooperative learning: Theory, research, and practice (2nd ed.), Boston: Allyn \& Bacon, 1995.

SPIRO R.J., JEHNG J.C. (1990). Cognitive flexibility and hypertext: Theory and technology for the nonlinear and multidimensional traversal of complex subject matter, In D. Nix, \& R.J. Spiro, Cognition, education and multimedia (pp. 163-205), Hillsdale, NJ: Erlbaum.

SPIRO R.J., FELTOVICH P.J., JACOBSON M.J., COULSON R.L. (1991). Cognitive flexibility, constructivism, and hypertext: Random access instruction for advanced knowledge acquisition in ill-structured domains, Educational Technology, Vol. 31 (May), pp. 24-33.

VADCARD L., LUENGO V. (2005). Réduire l'écart entre formations théorique et pratique en chirurgie : conception d'un EIAH. EIAH 2005. Montpellier, France (25-27 mai).

VAZQUEZ-ABAD J., BROUSSEAU N., WALDEGG C G., VÉZINA M., MARTÍNEZ DORADO A., PAUL VERJOVSKI J., CARVAJAL E., GUZMAN M.L. (2003). An approach to distributed collaborative science learning in a multicultural setting, In Constantinou, C. \& Zacharia, Z. C. (Eds.), Computer Based Learning in Science 2003, Volume I: New Technologies and Their Applications in Education, Nicosia: University of Cyprus

VYGOTSKY L.S. (1962). Thought and language, Cambridge, MA: MIT Press, 1962.

WALLON H. (1941). L'évolution psychologique de l'enfant, Paris: Armand Colin, 1941.

WebCT (2005). Web course tools, Retrieved December 24, 2005 from: http://www.webct.com.

WEBER G., BRUSILOVSKY P. (2001). ELM-ART: an adaptive versatile system for Web-based instruction, International Journal of Artificial Intelligence in Education, Vol. 12, pp. 351-384.

WILSON B.G. (1996). Constructivist learning environments: Case studies in instructional design, Englewood Cliffs, NJ: Educational Technology Publications, 1996.

WILSON B.G. (1997). Reflections on constructivism and instructional design, In C.R. Dills \& A.A. Romiszowski (Eds.), Instructional development paradigms, Englewood Cliffs NJ: Educational Technology Publications.

WINER L.R., RUSHBY N., VÁZQUEZ-ABAD J. (1999). Emerging trends in instructional interventions, In Stolovitch, H.S. et Keeps, E. (Eds.), Handbook of Human Performance Technology (2nd ed.) (pp. 884-886), San Francisco, Jossey-Bass.

WITTROCK M.C. (1985). Teaching learners generative strategies for enhancing reading comprehension, Theory into Practice, Vol. 24, pp. 123-126.

\section{A propos des auteurs}

Chieu VU MINH est actuellement chercheur postdoctoral du projet ThEMaT (Thought Experiments in Mathematics Teaching) à l'University of Michigan, School of Education. Avant de joindre ThEMaT, il était chercheur postdoctoral du projet TELEOS (Technology-Enhanced Learning Environment for Orthopedic Surgery) au laboratoire CLIPS du CNRS, Grenoble, France, où il a rédigé le présent article. Il s'intéresse aux technologies avancées pour favoriser l'enseignement et l'apprentissage, d'un point de vue constructiviste, en particulier pour stimuler et faciliter la flexibilité cognitive et la résolution de problèmes.

Adresse : 610 E. University Ave. \#534, Ann Arbor, MI-48109, United States of America

Courriel : vmchieu@umich.eduai

Toile : http://grip.umich.edu

Elie MILGROM est professeur émérite au Département d'Ingénierie informatique de l'Université catholique de Louvain (UCL). Il a consacré les dernières années de sa carrière à aborder une question que les premières années de cette même carrière lui ont fait apparaître comme cruciale pour tout enseignant-chercheur: que faire pour que nos étudiants apprennent effectivement ce que nous souhaitons qu'ils apprennent ? A ce titre, il a été un des promoteurs de l'introduction de l'apprentissage par problèmes et par projets à l'UCL et il a dirigé des recherches dans le domaine de l'utilisation des technologies au service de l'apprentissage.

Adresse : INGI, Place Sainte-Barbe, 2, B-1348 Louvain-la-Neuve

Courriel : Elie.Milgrom@uclouvain.be

Toile : $\underline{\text { http://www.info.ucl.ac.be/Bienvenue/presentation.php }}$ 
Référence de l'article :

Chieu VU MINH, Elie MILGROM, Vers une approche opérationnelle pour l'évaluation des EIAH, Revue STICEF, Volume 14, 2007, ISSN : 1764-7223, mis en ligne le 27/11/2007, http://sticef.org

(C) Revue Sciences et Technologies de 1'Information et de la Communication pour l'Éducation et la Formation, 2007 